Revista Mexicana de Astronomía y Astrofísica, Volumen 3, Mayo 1977

\title{
EFFETS DE SELECTION DANS LA DECOUVERTE DE SYSTEMES MULTIPLES
}

\author{
P. MULleR \\ CERGA, Grasse," France
}

\section{RESUMEN}

Estudiando algunos sistemas múltiples conocidos mediante un instrumento más poderoso, se hace ver que un gran número de estrellas dobles no había sido examinado anteriormente con tal instrumento, y se concluye que muchos de estos sistemas dobles son triples.

\section{ABSTRACT}

En étudiant les systèmes multiples issus d'un couple de découverte ancienne grâce à l'emploi ultérieur d'un instrument plus puissant, on constate qu'un grand nombre d'étoiles doubles n'ont jamais été examinées avec un tel instrument; il en résulte que parmi ces couples plusieurs centaines sont des systèmes au moins triples que nous ignorons.

La sélection intervient à tous les stades de notre connaissance des étoiles doubles et multiples: à la découverte, lors des observations, enfin au moment de calculer les orbites. Les facteurs les plus importants sont la puissance et la position géographique des instruments, les coordonnées des objets (et notamment leur distance polaire), les causes saisonnières, parfois certaines particularités des coupoles ou des instruments.

En ce qui concerne les systèmes multiples, on rencontre des types de sélection propres et j'ai étudié le problème de leur découverte en faisant la remarque suivante.

Ces systèmes sont apparus dans les catalogues selon deux mécanismes différents:

a) l'auteur de la découverte est unique, c'est-à-dire qu'il a trouvé en même temps, ou au moins dans le cours d'une même opération de recherche, les " $n$ " composantes, dont l'ensemble porte son nom;

b) un premier observateur a trouvé un couple, et par la suite un second plus habile ou mieux armé a constaté la présence d'une troisième composante (et éventuellement d'autres), qui avait échappé au premier parce qu'elle était trop faible ou trop proche de l'une des deux étoiles primitives.

Je me suis intéressé à cette découverte en deux temps, que j'appelle un "transfert", pour la raison qu'un grand nombre de couples actuellement catalogués ont été trouvés avec des insiruments moyens, ou par des observateurs qui pour quelque motif avaient une limite personnelle, et n'ont jamais été ensuite examinés dans des conditions nettement meilleures.

-Il fallait d'abord chercher à dénombrer ces transferts à partir d'un couple ancien, devenu plus tard multiple grâce à l'intervention d'un instrument plus grand, afin d'en évaluer la fréquence. Le problème est difficile pour beaucoup de raisons, et d'abord parce que jamais deux surveys successifs n'ont porté exactement sur le même choix d'étoiles; la comparaison la moins mauvaise et probablement la seule serait entre le survey de W. Struve $(\Sigma)$ et celui de Lick $(\mathrm{A}+\mathrm{Hu})$ et nous y reviendrons plus loin. Quand on parcourt l'ADS, qui contient pratiquement tous les couples anciens, on relève surtout des transferts-types entre $h, \Sigma$ ou $O \Sigma$ d'une part et $\beta$ ou Lick d'autre part, mais un certain nombre d'autres également. Dans un tel dénombrement, l'une des difficultés est d'éliminer autant que possible le cas où il $\mathrm{y}$ a des composantes optiques, soit dans le couple primitif, soit dans les étoiles découvertes par la suite. Il se trouve justement que certains observateurs ont ajouté presque systématiquement des composantes écartées aux couples anciens, par 
exemple Burnham et surtout Fox; ceci nullement dans la pensée de définir un système multiple, mais bien plutôt pour constituer un repère de mouvement propre possible donc en faisant une hypothèse exactement contraire. Dans les cas de ce genre, il est souvent impossible de décider si la composante lointaine est optique ou non, pour la simple raison que la première mesure est restée la seule, comme le faisait déjà remarquer W. D. Heintz en regrettant que ce genre de systèmes soient si négligés, car tous ne sont pas optiques. Les seuls cas à éliminer réellement, de ce point de vue, sont ceux oū la composante écartée était probablement à la portée du premier observateur, mais n'a pas mérité à ses yeux l'intérêt d'une mesure car ici il n'y a pas eu sélection dans le sens où nous l'entendons. Exemple:

$$
\begin{array}{rrr}
\text { ADS } 7885 \mathrm{AB}=\Sigma 1464 & 7,9-10,6 & 5,5 \\
-\mathrm{C} & (\beta) \quad-10 & 59^{\prime \prime 2}
\end{array}
$$

Malheureusement, les découvreurs notent rarement la présence d'une étoile supplémentaire s'ils ne l'ont pas mesurée.

En revanche, du seul point de vue de la sélection par la puissance de l'instrument, il n'importe pas finalement que l'une des composantes soit optique ou fasse partie au contraire du système.

J'ai donc négligé, en relevant ces transferts, les systèmes où la composante lointaine avait été ajoutée, alors qu'elle eût été, en apparence, mesurable dès la découverte de l'objet primitif; et aussi ceux où cette composante était vraiment trop lointaine pour présenter de l'intérêt. Il est évident que ce choix reste arbitraire, et difficile à traduire par des limites simples tant il intervient de paramètres.

Sous ces réserves inévitables, j'ai dénombré dans l'ADS, en tout, 380 de tels transferts; les plus fré-

\begin{tabular}{|c|c|c|}
\hline $\mathrm{h}$ & $\beta$ & 24 \\
\hline h & Lick & 30 \\
\hline$\Sigma$ & $\beta$ & 71 \\
\hline$\Sigma$ & Lick & 64 \\
\hline Os & $\beta$ & 12 \\
\hline $\mathrm{Oz}$ & Lick & $=16$ \\
\hline$\Sigma$ & $\mathrm{D}$ & 14 \\
\hline$\beta$ & Lick & 57 \\
\hline
\end{tabular}
quents sont les suivants:

cette liste représentant environ $60 \%$ du total.
Le nombre des couples anciens ( $h, \Sigma, O \Sigma$ ) est d'environ 3100 ; 250 transferts en gros les ont pour origine, soit sensiblement $8 \%$. Par conséquent, les couples découverts avec des instruments petits ou moyens se sont révélés, dans cette proportion, être des systèmes multiples dont l'existence nous était cachée par la sélection étudiée ici. Le problème posé est de savoir combien de couples actuellement connus ont échappé à un tel transfert.

Les couples anciens dans leur généralité sont des étoiles du $\mathrm{BD}$; la première question est de savoir s'ils ont tous été examinés à Lick, ou au moins par Burnham. Pour ce dernier, on sait que sa recherche n'a pas été systématique. A Lick en revanche on a suivi un programme bien défini qui comprenait le BD jusqu'aux étoiles de 9,0 inclusivement, plus tard étendu aux 9,1. Selon la fonction de magnitude, le reste $(9,2$ à 9,5$)$ devrait représenter $25 \%$ du $\mathrm{BD}$; en fait, un sondage par groupes de 100 dans tout le catalogue aboutit à constater un reste de $60 \%$ environ. La raison en est que beaucoup de 9,5 du $\mathrm{BD}$ sont en réalité des 9,6 ou des 9,7 et cela suffit à expliquer ces $60 \%$. D'autre part, il semble qu'à Lick on ne se soit pas limité de façon rigide à 9,1 ; on trouve parmi les $\mathrm{A}$ et les $\mathrm{Hu}$ un certain nombre de couples issus d'étoiles plus faibles. On peut donc admettre qu'en principe toutes les étoiles du BD qui se trouvent dans l'ADS ont été examinées à Lick; ce sera en tout cas un maximum, et le reste, c'est-à-dire le nombre de celles qui ne l'ont pas été, représentera un minimum de la réalité. Un sondage dans l'ADS montre que les étoiles du BD y sont environ $87 \%$; le reste est donc de $13 \%$ soit en gros 2200 étoiles. A ce nombre il faut ajouter celui des couples découverts après le survey de Lick, c'està-dire en pratique hors de l'ADS. Entre les grandes listes des découvertes d'étoiles faibles on a retenu les J, Es, Mlb et Stein.

Dans le cas des J, l'auteur a donné heureusement une liste de celles qui sont identifiées dans le BD. Sachant par ailleurs que l'ADS s'arrête à J 1358, il est facile de relever les découvertes ultérieures non BD qui sont au nombre de 1500. Dans les autres listes, on trouve après l'ADS $600 \mathrm{Mlb}, 1900$ Stein et $500 \mathrm{Es}$; la proportion des étoiles du BD y est très variable: $50 \%$ pour les Mlb, très peu pour les Stein, 80 à $90 \%$ pour les Es. Le reste pour ces trois listes représente donc environ, 2000 à 2500 objets. 
Avec les étoiles non BD de l'ADS, on constate donc que près de 5500 couples actuellement connus n'ont jamais été examinés avec un instrument de grande ouverture lors d'un survey systématique. Ceci n'exclut pas les découvertes de hasard, à l'occasion de mesures courantes aux grands instruments mais les conditions moyennes d'observation ne sont pas comparables à celles que l'on exige d'un survey et ces découvertes restent rares. Si la fréquence des transferts relevée dans les cas réels relevés au début, soit $8 \%$, est valable, ce sont donc au moins 400 systèmes multiples, et probablement beaucoup plus, que nous ignorons par le seul effet de la sélection étudiée ici.
Il importe de souligner qu'une faible partie seulement de ces systèmes inconnus sont susceptibles de découverte, car ils correspondent à un transfert à partir de couples généralement faibles eux-mêmes, contrairement aux transferts relevés dans l'ADS qui avaient pour origine des $\Sigma, O \Sigma$ ou h; dans la majorité des cas, les compagnons supplémentaires seront cette fois soit beaucoup trop rapprochés d'une composante, soit trop faibles pour être observés. C'est ce qui explique la proportion de tels transferts lors des recherches de $P$. Couteau et de moi-même, et qui n'est que de $2 \%$ des couples déjà connus et non de $8 \%$ comme dans l'ADS.

\section{DISCUSSION}

Couteau: Dans les prospections actuelles à Nice on trouve $2 \%$ de triples parmi les couples déjà connus. Ce sont indifféremment les primaires ou les secondaires qui sont doubles.

Muller: Il est normal que l'on trouve $2 \%$ seulement et non $8 \%$ car la population moyenne explorée est en faite d'objets plus faibles que les couples anciens sur lesquels on a constaté $8 \%$ de transferts.

Poveda: I find a contradiction between your $8 \%$ proportion of triple stars among $\mathrm{h}-$, $\Sigma-$, and $\mathrm{O} \Sigma$ binaries and the $20 \%$ which Worley just reported. Will you please comment on this?

Muller: The $8 \%$ proportion concerns transfers whose origin is ancient and of generally easy pairs. If we start with a new sample of stars like those of Es, Mlb, Stein, and J, most of the resulting triples will not be observable.

Worley: I stated that my statistics included spectroscopic, astrometric, etc. systems.

King: It seems to me that much work on common proper motions remains to be done, to re-examine stars systematically for proper motions smaller than the limit of the surveys for large proper motions.

Muller: You are certainly right, and a resolution should perhaps be proposed.

Scarfe: About how many of the very wide systems have been examined to test whether they do show common proper motion?

Muller: I have no information about that.

Strand: The Luyten and Lowell proper motion programs were designed to find stars with proper motions larger than a given amount $(0.20$ arcsec $)$. These programs would therefore not necessarily measure common proper motions of distant companions of binaries.

van de Kamp: Distant proper motion companions have been and are being found to single stars. I suggest that double star observers pay attention to the, as yet, single components of wide proper motion doubles in the hope of resolving such components, one or both, into close binaries (visual and/or spectroscopic).

\section{GENERAL DISCUSSION}

Worley: There appears to be a strong selection effect in the occultation observations caused (perhaps deliberately) by the exclusion of possible wider pairs $\rho \sim 0.5-5$ arcsec. I suspect many such systems exist and could be detected by this method.

Evans: Yes, here is a selection in favor of very close pairs. The occultation method is particularly valuable for resolution of close and multiple stars, and so there is a concentration on these. In very many cases of known visual binaries the components are so far apart that they are best observed separately. One cannot use too large a diaphragm because of excessive moonlight. We retain 512 observational points after the automatic cutoff. I agree that we must be losing some wide pairs because of data limitations, but we have to compromise because of practical considerations. 